\title{
Intrauterine Device Transmigration Into Sigmoid Bowel in Early Pregnancy
}

\author{
Lauren Schiff, MD \\ Women's Health Services, Department of Obstetrics and Gynecology, Henry Ford Health System, Detroit, MI, USA.
}

\begin{abstract}
Introduction: Uterine perforation at the time of intrauterine device (IUD) insertion is estimated to occur at a rate of 1 per 1000 insertions. Most published cases describing transmigration into abdominal viscera are in the setting of uterine perforation. In the case described here, IUD transmigration into the bowel lumen is chronologically documented in early pregnancy and apparently unrelated to uterine perforation.

Case Description: A 36-year-old gravida 7, para 7 woman seeking permanent sterilization explained that she became pregnant with her last child with a copper IUD in place. The device was intrauterine on her first obstetric ultrasonogram at 6 weeks, 2 days of gestation. Subsequent ultrasonography 2 days later revealed no intrauterine IUD. The patient had a successful full-term delivery. Four months following delivery, the IUD was found on an abdominal X-ray at the level of the sacrum. On laparoscopic evaluation, the IUD was found embedded in the lumen of the sigmoid colon with only the strings visibly protruding through the serosa.

Discussion: The rates of bowel injury related to IUD insertion and delayed translocation are unknown. The mechanism of translocation is not fully understood. A possible mechanism may be partial- to full-thickness myometrial penetration at the time of insertion allowing fistualization between the uterus and a hollow viscus via inflammatory processes. The documented early pregnancy translocation of an IUD suggests that intrauterine pregnancy may be the impetus for this process and reinforces the recommendation to remove an intrauterine IUD in pregnancy at the time of diagnosis.
\end{abstract}

Key Words: Intrauterine device, Laparoscopy, Perforation, Pregnancy, Sigmoid colon.

Citation Schiff L. Intrauterine device transmigration into sigmoid bowel in early pregnancy. CRSLS e2014.00164. DOI: 10.4293/CRSLS.2014.00164.

Copyright (c) 2014 SLS This is an open-access article distributed under the terms of the Creative Commons Attribution-Noncommercial-ShareAlike 3.0 Unported license, which permits unrestricted noncommercial use, distribution, and reproduction in any medium, provided the original author and source are credited.

Address correspondence to: Lauren Schiff, MD, Henry Ford Hospital, Women's Health Services, 6777 W Maple Rd, West Bloomfield, MI, 48322. Telephone: 617-699-6672, Fax: 248-325-0094, E-mail: schiff.lauren@gmail.com

\section{INTRODUCTION}

Uterine perforation at the time of intrauterine device (IUD) insertion is estimated to occur at a rate of 1 per 1000 insertions. ${ }^{1}$ Extrauterine migration of an IUD following perforation is reported in case studies, but it remains a relatively rare occurrence. Estimating the incidence of this event is challenging because cases may go undetected for a prolonged time following insertion.

IUDs found outside the uterus are thought to be due to partial or complete uterine perforation at the time of insertion. These cases most commonly result in an IUD that is found in the intraperitoneal cavity adherent to omentum and/or bowel, involved in a tubo-ovarian abscess, or freely floating in the peritoneal cavity. ${ }^{2}$ Bowel perforation is more rare; however, case reports of true bowel perforation and intraluminal transmigration have been published, with the first notable case reported as early as $1981 .{ }^{3}$ Cases of IUD bowel perforation are most commonly reported as abdominopelvic pain and the absence of visible IUD strings.

Most cases of bowel translocation reported involve the copper IUD, suggesting the importance of the inflammatory nature of the copper to incite transmigration into the bowel. Key and Kreutner ${ }^{4}$ suggested in 1980 that the underlying mechanism was pressure necrosis followed by perforation into a hollow viscus, which allowed the migration of the IUD over a prolonged period. Alternatively, the IUD may enter directly into the sigmoid or rectum at 
the time of placement due to use of excessive force on insertion. ${ }^{4,5}$

The extrauterine or "missing" IUD is commonly identified during pregnancy. It is hypothesized that this may be due to malplacement and partial perforation at the time of insertion, progressing to complete translocation during pregnancy. We present a case of an intraluminal copper IUD found in the sigmoid several months after insertion. Serial ultrasonograms suggest early pregnancy to be the stimulus for translocation, rather than perforation at the time of insertion. Treatment required bowel resection due to intraluminal and full-thickness bowel fibrotic reaction caused by the copper IUD.

\section{CASE}

A 36-year-old gravida 7, para 7 woman presented to the general gynecology clinic seeking permanent sterilization with (Bayer Health Care Pharmaceuticals [Whippany, NJ/ USA]) inserts. During her interview, she revealed that she became pregnant with her last child with an IUD in place, and the device was seen to be intrauterine on her first obstetric ultrasonogram. She reported not knowing if she passed the IUD vaginally at delivery and was unaware of whether it was currently in situ. On speculum examination, no IUD strings were visualized.

On review of her obstetric ultrasonograms, an IUD was seen via ultrasonography taken at 6 weeks, 2 days and reported to be "posterior and slightly to the right of the gestational sac, within the endometrial cavity." A normal fetal heartbeat was present. Transvaginal ultrasonography was repeated 2 days later due to the onset of vaginal spotting, and the IUD was reported to not be visualized. This absence of an intrauterine IUD was confirmed on both 21-week and 37-week ultrasonograms. The patient delivered at full term via normal spontaneous vaginal delivery with no documentation of passage of the IUD. No further imaging was performed postpartum.

Pelvic ultrasonography was performed as part of the preoperative evaluation for the Essure inserts and demonstrated no intrauterine IUD. Abdominal X-ray showed an IUD at the level of the sacrum. The decision was made to pursue permanent sterilization via laparoscopic tubal ligation with concomitant retrieval of the intraperitoneal IUD. The patient understood that retrieval of the IUD could involve additional procedures involving her bowel, bladder, the uterus, and tubes, including but not limited to hysterectomy, oophorectomy, salpingectomy, and bowel resection. The patient consented to undergo all necessary procedures for removal of her IUD.

Intraoperatively, a uterine manipulator was placed. Laparoscopic entry was obtained infraumbilically. Additional lateral ports were placed to aid in bowel retraction for locating the IUD. A thick fibrotic adhesion was noted that extended from the posterior uterus to the sigmoid and was surrounded by thin filmy adhesions. These thin adhesions were transected revealing the IUD strings protruding from the lumen of the sigmoid at the base of the thick adhesion to the sigmoid (Figure 1). Fibrotic tissue was noted on the serosal surface of the sigmoid. Rectal examination failed to retrieve the IUD, due to its proximal location. A colorectal surgery consult was obtained and flexible diagnostic sigmoidoscopy was performed demonstrating both arms of the IUD to be intraluminal with one arm transmu$\mathrm{ral}$ and adherent to the mucosal epithelium of the sigmoid colon. Because of the IUD's transmural location, the decision was made by the colorectal team to perform a laparoscopic rectosigmoid colonic resection with primary anastomosis (Figure 2). Minilaparotomy with placement of a GelPort system was performed to complete the resection with the Endo GIA stapler (Covidien Surgical [Mansfield, MA/USA]). The anastomosis was completed using the EEA stapler (Covidien Surgical [Mansfield, MA/ USA]) with transanal approach. The leak test was negative.

Notably, tubal ligation with Filshie clips was performed prior to bowel resection. The patient tolerated the surgery well and was discharged home on postoperative day 3 passing flatus and bowel movements. The patient

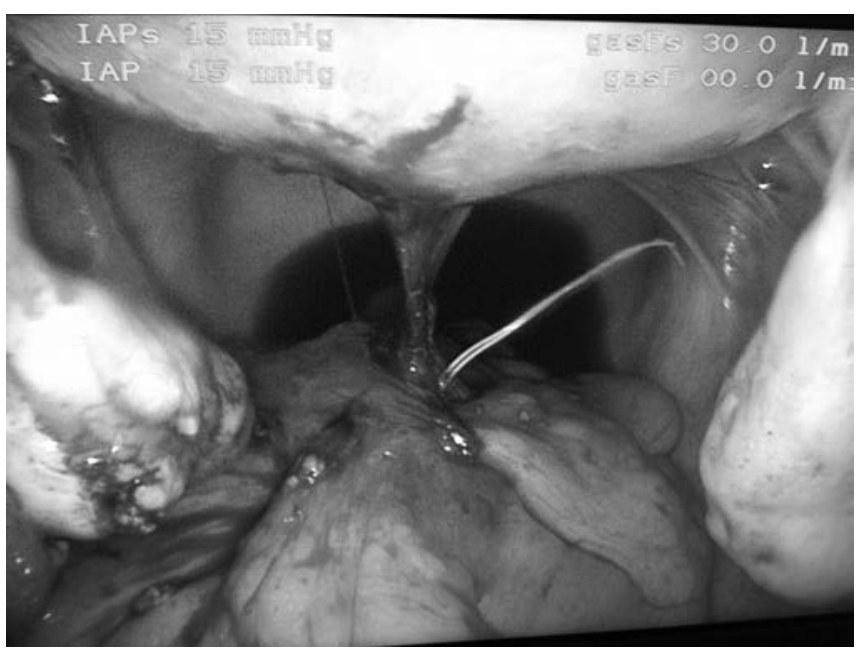

Figure 1. IUD strings protruding from the lumen of the sigmoid at the base of the thick adhesion to the uterus. 


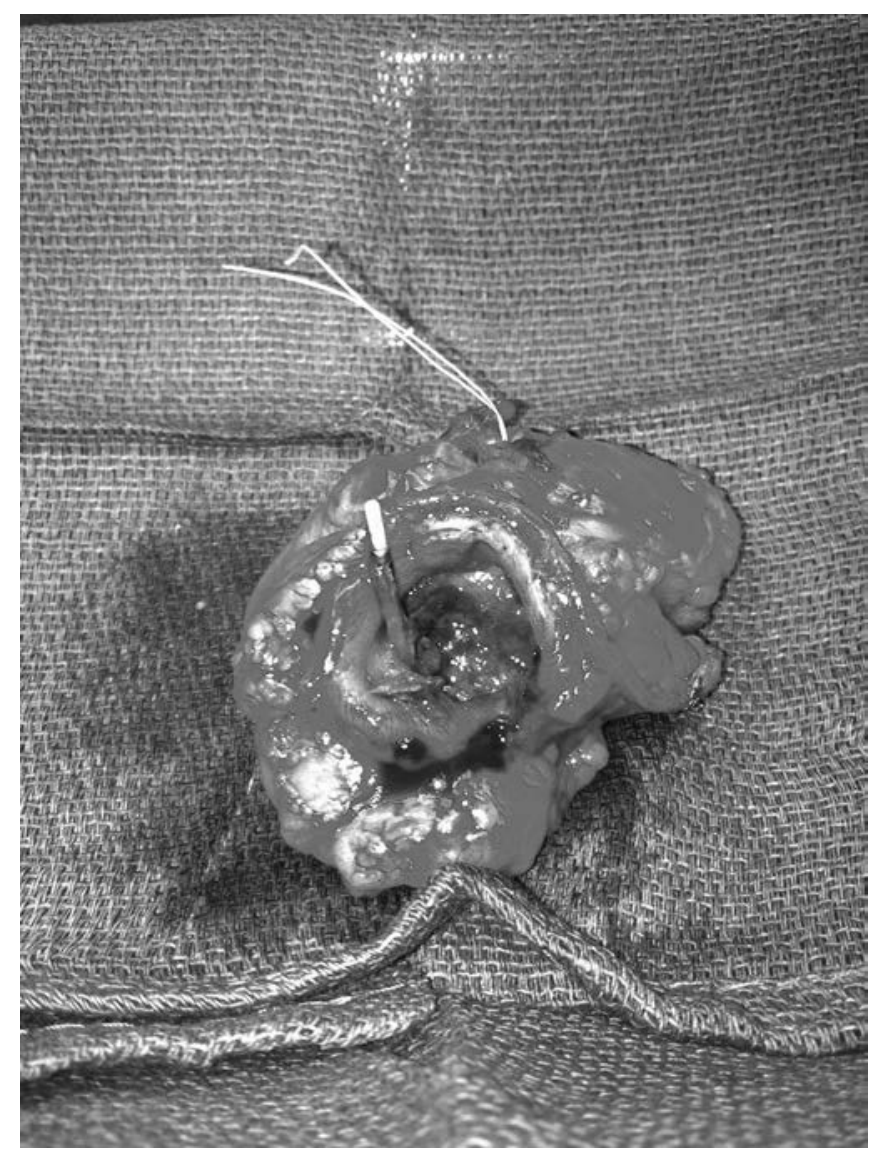

Figure 2. Segment of resected rectosigmoid containing IUD. One arm of IUD transmural and IUD strings protruding through serosal surface.

has done very well postoperatively without further complaints.

\section{DISCUSSION}

The IUD is the most commonly used reversible contraception worldwide. ${ }^{6}$ In the United States, its use has been increasing in recent years. ${ }^{7}$ Patients are counseled prior to insertion regarding the risk of perforation, which is described at a rate of 1 in $1000 .{ }^{1}$ The rates of bowel injury related to insertion and/or delayed translocation are unknown. Bowel perforation at the time of insertion may be suspected with difficult placement and greater than expected patient discomfort. ${ }^{8}$ Unfortunately, IUD placement is a particularly uncomfortable procedure for many patients, and evaluation of subjective patient pain during this procedure cannot be relied on to indicate that postinsertion ultrasonographic evaluation of placement is necessary.
The likelihood that true bowel perforation occurs at the time of placement is low given the lack of case reports of bowel leakage, sepsis, bowel abscess, and similar conditions. Review of the case reports supports Key and Kreutner's initial theory-that partial- to full-thickness myometrial penetration at the time of insertion allows for what is essentially fistualization between the uterus and a hollow viscus via inflammatory processes. This was likely the etiology in a case described by Taras and Kaufman, ${ }^{9}$ in which pregnancy occurred 9 months after the initial IUD placement, resulting in spontaneous abortion and requiring dilation and curettage for retained products of conception. In this case, pregnancy may have occurred due to initial IUD malplacement with transmigration incited by pregnancy and uterine instrumentation. Delayed transmigration in the setting of pregnancy may occur even when correct IUD placement is assumed due to prolonged successful contraception. Glass et $\mathrm{al}^{10}$ described such a patient who became pregnant 9 years after IUD placement. The IUD was seen on consecutive ultrasonograms to travel from the lower uterine segment in first trimester, embed in the fundal myometrium in the second trimester, and migrate into the sigmoid colon by 6 weeks postpartum.

Similar to that of Glass et $a l,{ }^{10}$ in the case presented here, the presence of an intrauterine pregnancy alone appears to be the impetus for this inflammatory transmigration process to occur. It may be surmised that the IUD was incorrectly placed initially, with partial intramural penetration (although not described as such on initial dating ultrasonogram), thus allowing for pregnancy. However, it was not the growth of the pregnancy that forced the IUD out of the uterus proper, given that "disappearance" of the IUD occurred between 6 weeks, 2 days and 6 weeks, 4 days of gestation. This suggests some inflammatory-mediated fistualization. This hypothesis is supported by the appearance of a thick fibrotic band attaching the posterior uterus to the sigmoid colon at the point of the IUD strings. No clear point of uterine perforation could be identified aside from this thick fibrous band. A slow process of fistualization and transmisgration is also suggested by the lack of pain on the part of the patient. Her initial presentation was purely for sterilization, and the missing IUD was an incidental finding. She had no bowel symptoms, and no significant pelvic pain was documented. The suggestion that the copper IUD interacts significantly with pregnancy is supported by 2 studies (1 prospective cohort and 1 case-control) demonstrating that the copper IUD was more likely to be malpo- 
sitioned in the setting of pregnancy versus nonpregnant control subjects. Whether malposition of the copper IUD led to pregnancy versus pregnancy leading to malposition has not been established. ${ }^{11,12}$

Removal of an IUD in early pregnancy is recommended. Women who retain a copper IUD during pregnancy risk increased rates of spontaneous abortion, premature rupture of membranes, preterm delivery, and chorioamnionitis. ${ }^{4}$ In the case presented here, the patient completed a successful pregnancy to term, perhaps because the IUD self-extravasated at an early gestation.

While prior authors have questioned the necessity of removal of an intraperitoneal copper IUD in asymptomatic patients, ${ }^{13}$ reports of colonic fistula creation, ${ }^{14}$ bowel perforation, ${ }^{7,15-21}$ tubo-ovarian abscess formation, ${ }^{18}$ and ovarian embedment ${ }^{17}$ argue for the prompt removal at diagnosis to avoid such sequelae. ${ }^{18}$ Localization and prompt removal of an extrauterine IUD is recommended to avoid hollow viscous perforation..$^{9,10}$ Prior to the advancement of laparoscopic techniques, this was most commonly managed with laparotomy. The more recent case reports have described laparoscopic removal using intentional enterotomy, ${ }^{19}$ laparoscopic bowel resection with primary anastomosis, ${ }^{22}$ and retrieval transanally with colonoscopy or proctosigmoidoscopy. ${ }^{23}$ Zeino et $\mathrm{al}^{20}$ have recently described success with laparoscopic colotomy for retrieval of a sigmoid IUD. In the case presented here, flexible sigmoidoscopy identified the IUD to be intraluminal with partial intramural location of one of the arms and fibrotic changes of the bowel wall. Laparoscopic evidence of full-thickness degraded tissue quality of the bowel wall extending $>1 \mathrm{~cm}$ in diameter raised concerns for appropriate healing and indicated bowel resection rather than colotomy or transanal retrieval. Laparoscopic bowel resection with primary anastomosis in this setting has been previously recommended by Chi et al. ${ }^{21}$ The involvement of colorectal surgeons to evaluate the viability of the surrounding bowel is advisable to provide the safest retrieval method.

In summary, early pregnancy may be a stimulus for translocation of an intrauterine copper IUD, and fibrotic fistualization may be a form of slow embedment into the sigmoid colon. Avoiding delays in locating and removing the IUD may decrease the risk for bowel complications. The decision to pursue intra-abdominal surgical retrieval during pregnancy should be made after weighing the risks and benefits to the mother and fetus. A systematic review of the literature to assess the rate of viscous perforation from the time of diagnosis of ectopic IUD is needed to guide this risk assessment. Cotreatment with colorectal surgery is recommended for adequate bowel evaluation, as simple retrieval via laparoscopy or colonoscopy may not be possible.

\section{References:}

1. Mechanism of action, safety and efficacy of intrauterine devices: report of a WHO Scientific Group. World Health Organ Tech Rep Ser. 1987;753:1-91.

2. Stuckey A, Dutreil P, Aspuru E, Nolan TE. Symptomatic cecal perforation by an intrauterine device with appendectomy removal. Obstet Gynecol. 2005;105(5 Pt 2):1239-1241.

3. Beard RJ. Unusual presentation of translocated intrauterine contraceptive device. Lancet. 1981;1(8224):837.

4. Key TC, Kreutner AK. Gastrointestinal complications of modern intrauterine devices. Obstet Gynecol. 1980;55(2):239244 .

5. Nohuz E, Battista R, Tamburro S, et al. A more and more painful intrauterine device ... where it is not enough to see the wires to exclude malposition! [in French]. Gynecol Obstet Fertil. 2012 Apr 19. [E-pub ahead of print].

6. Mosher WD, Martinez GM, Chandra A, Abma JC, Willson SJ. Use of contraception and use of family planning services in the United States: 1982-2002. Adv Data. 2004;(350):1-6.

7. American College of Obstetricians and Gynecologists. ACOG Practice Bulletin No. 121: long acting reversible contraception: implants and intrauterine devices. Obstet Gyencol. 2011;118(1):184-196.

8. Kuś E, Swierczewski A, Pasiński J, Estemberg D, et al. Intrauterine contraceptive device in an appendix-a case report [in Polish]. Ginekol Pol. 2012;83(2):132-135.

9. Taras AR, Kaufman JA. Laparoscopic retrieval of intrauterine device perforating the sigmoid colon. JSLS. 2010;14(3):453-455.

10. Glass T, Baker T, Kauffman RP. Migration of an intrauterine contraceptive device during the course of pregnancy: a case report. J Minim Invasive Gynecol. 2009;16(1):81-83.

11. Inal MM, Ertopçu K, Ozelmas I. The evaluation of 318 intrauterine pregnancy cases with an intrauterine device. Eur J Contracept Reprod Health Care. 2005;10(4):266-271.

12. Pakarinen $\mathrm{P}$, Luukkainen T. Five years' experience with a small intracervical/intrauterine levonorgestrel-releasing device. Contraception. 2005;72(5):342-325.

13. Markovitch O, Klein Z, Gidoni Y, Holzinger M, Beyth Y. Extrauterine mislocated IUD: is surgical removal mandatory? Contraception. 2002;66(2):105-108. 
14. Pirwany IR, Boddy K. Colocolic fistula caused by a previously inserted intrauterine device: case report. Contraception. 1997;56(5):337-339.

15. McWhinney NA, Jarrett R. Uterine perforation by a Copper 7 intrauterine contraceptive device with subsequent penetration of the appendix: Case report. Br J Obstet Gynaecol. 1983;90(8):774-776.

16. Bitterman A, Lefel O, Segev Y, Lavie O. Laparoscopic removal of an intrauterine device following colon perforation. JSLS. 2010;14(3):456-458.

17. Verma U, Verma N. Ovarian embedding of a transmigrated intrauterine device: a case report and literature review. Arch Gynecol Obstet. 2009;280(2):275-278.

18. Mederos R, Humaran L, Minervini D. Surgical removal of an intrauterine device perforating the sigmoid colon: a case report. Int J Surg. 2008;6(6):e60-62.
19. Ingec M, Kumtepe Y, Kadanali S, Ozdiller O. A rare case of ileal embedding by an intrauterine device. Eur J Contracept Reprod Health Care. 2005;10(1):29-31.

20. Zeino MY, Wietfeldt ED, Advani V, Ahad S, Younkin C, Hassan I. Laparoscopic removal of a copper intrauterine device from the sigmoid colon. JSLS. 2011;15(4):568-570.

21. Chi E, Rosenfeld D, Sokol TP. Laparoscopic removal of an intrauterine device perforating the sigmoid colon: a case report and review of the literature. Am Surg. 2005;71(12):1055-1057.

22. Chen CP, Hsu TC, Wang W. Ileal penetration by a Multiload-Cu 375 intrauterine contraceptive device: a case report with review of the literature. Contraception. 1998;58(5):295-304.

23. Medina TM, Hill DA, DeJesus S, Hoover F. IUD removal with colonoscopy: a case report. J Reprod Med. 2005;50(7):547-549. 